\title{
Efficacy of Mesenchymal Stromal Cells in Preclinical Models of Necrotizing Enterocolitis: a Systematic Review Protocol
}

Camille Maltais-Bilodeau ( $\sim$ cmaltaisbilodeau@cheo.on.ca )

Ottawa University https://orcid.org/0000-0001-7534-6813

Ewa Henckel

Karolinska Institutet

Kelly D. Cobey

Ottawa Hospital Research Institute

Nadera Ahmadzai

Ottawa Hospital Research Institute

\section{Becky Skidmore}

Ottawa Hospital Research Institute

\section{Emanuela Ferretti}

Ottawa General Hospital: Ottawa Hospital

\section{Bernard Thébaud}

Ottawa Hospital Research Institute

\section{Protocol}

Keywords: necrotizing enterocolitis, neonatal enterocolitis, mesenchymal stromal cells, stem cells, preclinical, animal models, systematic review protocol

Posted Date: February 23rd, 2021

DOI: https://doi.org/10.21203/rs.3.rs-239448/v1

License: (c) (1) This work is licensed under a Creative Commons Attribution 4.0 International License. Read Full License

Version of Record: A version of this preprint was published at F1000Research on October 5th, 2021. See the published version at https://doi.org/10.12688/f1000research.73094.1. 


\section{Abstract}

\section{Background}

Necrotizing enterocolitis is an acute inflammatory disease of the intestine that can lead to necrosis and bowel perforation. It is a severe complication of preterm birth with a prevalence of $7 \%$ in infants with a birth weight less than 1500 grams. It's mortality rate is up to $30 \%$ and survival after necrotizing enterocolitis leads to long-term gastrointestinal and neurocognitive consequences. The current treatment is supportive and includes bowel rest and decompression and antibiotics. Thus, new treatments are necessary to reduce mortality and morbidity. Mesenchymal stromal cells are known to have antiinflammatory properties and might be a promising option for treatment. Here we present a protocol for a systematic review with the aim to explore the efficacy of cell therapies with mesenchymal stromal cells in animal models of necrotizing enterocolitis. The primary outcome is histological signs of necrotizing enterocolitis. Additional outcomes include survival, bowel perforation, gut permeability, gut motility, levels of inflammatory markers, cytokine levels and adverse events.

\section{Methods}

We will conduct a systematic search of MEDLINE, Embase, and Web of Science databases. The retrieved records will be screened individually by two investigators. We will include all preclinical in vivo animal models of experimentally induced NEC that evaluate the efficacy of mesenchymal stromal cells or other cell therapy treatments. Outcome data will be extracted from each article and risk of bias assessment performed. Funnel plots and SYRCLE's risk of bias tool for animal studies will be used. Data will be reported as ratios, divided in predefined subgroups where relevant.

\section{Discussion}

This systematic review aims to examine the efficacy of mesenchymal stromal cells in preclinical models of necrotizing enterocolitis and whether there is sufficient evidence to support a clinical trial of efficacy and safety of the treatment with mesenchymal stromal cells in infants with necrotizing enterocolitis.

\section{Systematic review registration}

This protocol has been registered on Open Science framework: osf.io/5rc6t

\section{Background}

Necrotizing enterocolitis (NEC) is an acute inflammatory disease of the intestine $(1,2)$. It was first described by Mizrahi et al. in 1965 (3). This disease is characterized by inflammation and injury to the wall barrier of the intestine that can cause necrosis and that can lead to bowel perforation $(2,4,5)$. It is a major complication of preterm birth $(6,7)$. In Canada, the preterm birth rate is $7.7 \%(8)$ and, on the basis of large databases, the mean prevalence of NEC in Canada and the United States is about $7 \%$ among infants with birth weight between $500 \mathrm{~g}$ and $1500 \mathrm{~g}(1,9,10)$ with a mortality rate as high as $30 \%(11)$. 
The pathogenesis of NEC is multifactorial (12). The most common known risk factors of NEC include low gestational age, low birth weight, exposure to infant formula-feeding and microbial dysbiosis $(2,4,12$, 13). The classic NEC pathophysiological process of intestinal injury appears to be related to a basic immaturity of host defences, culminating in an excessive inflammatory response, with serum cytokines and chemokines, especially IL-8 significantly elevated (14-16) and imbalance of the healthy microbiome.

Survivors of a NEC episode are at increased risk of long-term adverse outcomes including gastrointestinal and neurodevelopmental complications(4). Because the disease occurs during an important time of brain development, the long-term NEC-associated neurodevelopment outcomes include cerebral palsy and visual, cognitive and psychomotor impairment $(4,17-22)$. NEC also significantly increases hospital length of stay and health care costs. Infants with NEC needing medical treatment are hospitalized on average 22 days longer in the neonatal intensive care unit than neonates without NEC, and those who require surgical treatment are hospitalized 60 days longer than infants without NEC (23). In the USA, the estimated average cost for medical and surgical treatment of NEC is $\$ 500000$ per patient $(23,24)$.

Current treatment strategies depend on the severity of the disease and remain supportive only. They include bowel rest and decompression, fluid support, vasopressors and systemic broad-spectrumantibiotics $(4,12,25)$. Surgical treatment of NEC is recommended if bowel perforation occurs or in some cases of bowel necrosis (12). In order to reduce the morbidity and mortality associated with this inflammatory disease, evaluation of new therapies is required. Several treatments have been studied in preclinical trials, including the utilization of mesenchymal stromal cells (MSCs).

MSCs were described in 1976 as fibroblast precursor cells with a role in the regulation of murine hematopoietic stem cells in bone marrow (26). Following this, multiple preclinical studies have shown a repair ability of MSCs in multiple injured organs and tissues of neonatal models such as in the brain (27$31)$, heart $(32)$, lungs $(33,34)$ gut $(35,36)$ and eyes $(37)$. MSCs are known to have anti-inflammatory properties (38) and considering that inflammation plays a key role in the pathogenesis of NEC, they may represent a promising therapeutic option for this disease.

\section{Objectives}

We aim to conduct a systematic review of the benefits and harms of MSC therapy in preclinical models of NEC. We seek to look for any clinical effect of this therapy in animal models. We will specifically address the question: In controlled preclinical studies of necrotizing enterocolitis, can MSCs reduce the histological signs of NEC in NEC-induced animal models? Our population of interest is all animal studies of NEC and we are interested in any such studies that use MSCs or any other cell therapy as an intervention. We are interested in all comparisons in the literature. Our primary outcome is histology showing NEC. The following additional outcomes will be measured: survival, bowel perforation, gut permeability, gut motility, levels of inflammatory markers, cytokine levels and adverse events.

\section{Methods}


This study protocol was developed through discussion between our research team which includes clinical and preclinical research experts (CMB, EH, BT, EF), an information specialist (BS), and research methodologists with expertise in knowledge synthesis (NA, KDC). The protocol was prepared using the PRISMA-P reporting guidelines (39) and the protocol format for the preparation, registration and publication of systematic reviews of animal intervention studies published by de Vries et al (40). This protocol was registered using the Open Science Framework (osf.io/5rc6t). A completed PRISMA-P checklist for this protocol is provided as an additional file (see additional file 1). Post-hoc modifications to the current protocol incurred during the review process will be documented in the publication of the final report to ensure transparency.

\section{Eligibility criteria}

We will include all study designs of research describing preclinical NEC models that evaluate the efficacy of MSCs or other cell therapy treatments that are published in English. Potentially relevant non-English materials will be provided in the appendix section but will not be included in the analysis. We will not limit our eligibility criteria by year of publication or publication status. Details on the study PICOS are provided below.

\section{Population}

The population will include all preclinical in vivo animal models of experimentally induced NEC. To enhance the generalizability of our findings, NEC will be defined as any histological intestinal tissue injury. Various mechanisms of NEC have been reported in animal models in the literature $(41,42)$. These include gavage formula or hyperosmolar formula gavage feeds, exposure to hypoxia and/or hypothermia and administration of bacteria.

\section{Intervention}

The intervention of interest is all MSCs cell types, independent of the source, in animal models. MSCs will be defined by using the International Society for Cellular Therapy consensus statement. The minimal criteria to meet to define human MSC are: (1) adhesion to plastic; (2) expression of the cell surface markers CD105, CD73 and CD 90 and lack of CD45, CD34, CD14 or CD11b, CD79ه or CD19 and HLA-DR surface molecules expression and (3) differentiation to osteoblasts, adipocytes and chondroblasts in vitro (43).

All types of MSCs will be considered in the review to enhance the generalizability of our study findings. Any therapeutic MSCs regimen (prophylactic - before or during induction of NEC, or rescue after induction of NEC) will be considered.

\section{Comparison}

In our population study of experimental animal models of NEC, we will consider all types of reported comparator. We will also consider groups with no comparator. 
Outcome

The primary outcome is histological signs of NEC measured at any time after the administration of MSCs. The secondary outcomes measured are: survival, bowel perforation, gut permeability (e.g. disruption of thigh junction proteins, liver fatty acid-binding protein level and intestinal fatty acid binding protein level), gut motility, levels of inflammatory markers (e.g. white blood cells count, C-reactive protein level and fecal calprotectine) cytokine levels (e.g., interleukine-8 and toll-like receptor 4) and any describe adverse events.

Study design

We will include any study design in the search including both comparative and non-comparative studies as well as empirical research.

\section{Information sources}

Search strategy

The search strategies will be developed and tested through an iterative process by an experienced medical information specialist in collaboration with the study team members. The MEDLINE strategy will be peer-reviewed by another senior information specialist prior to execution using the Peer Review of Electronic Search Strategies (PRESS) guidelines (44). Proposed databases include MEDLINE, Embase, and Web of Science. Searches will utilize a combination of controlled vocabulary (e.g., "Stem cells", "Mesenchymal Stem Cells", "Stromal Cells" and "Enterocolitis, Necrotizing") and keywords (e.g., "MSCs", "stromal cell", "neonatal enterocolitis"). Vocabulary and syntax will be adjusted across the databases. To enhance search efficacy, we will use animal filters validated for Pubmed/MEDLINE and Embase (40, 45, 46), amended as appropriate to accommodate new developments in vocabulary. There will be no language or date restrictions on any of the searches. We will perform a grey literature search of conference websites and animal research organizations' websites to identify literature not included in the aforementioned databases, and also perform general searches using Google Scholar.

\section{Study records}

\section{Data management}

DistillerSR (Evidence Partners Inc, Ottawa, Canada) software will be used for records and data management throughout the review. The resulting search citations will be deduplicated and then imported into DistillerSR. Study screening and data extraction forms will be created on the platform to facilitate review of citations obtained from the search.

\section{Selection process}

Two reviewers, independently and in duplicate, will carry out citation screening in two stages, reviewing records against the a priori eligibility criteria using an online systematic review software program (Distiller 
Systematic Review (DSR) Software; Evidence Partners Inc, Ottawa, Canada). At Stage 1, the titles and abstracts of all records obtained from the search will be screened in duplicate independently by CMB and EH while Stage 2 screening will encompass full-text review of the articles deemed potentially relevant at Stage 1. If there are disagreements between the reviewers, it will be resolved by a consensus or by a third member of the study team (EF or BT). Both stages of screening will begin with a calibration exercise to ensure consistent application of eligibility criteria between reviewers. This will include pilot screening of titles and 25 citations at Stage 1 and 25 full-text articles at Stage 2. In accordance with the Preferred Reporting Items for Systematic Reviews and Meta-Analyses (PRISMA) guidelines (39), the exclusion reasons of potentially eligible studies will be recorded.

Data collection process

The two independent investigators ( $\mathrm{CMB}$ and $\mathrm{EH})$ conducting screening and extraction will use standardized forms (See drafts in Appendix 1). These forms will be piloted on a set of 10 documents and then discussed. If necessary, changes to the forms will be made and they will be re-piloted on 10 further documents. This process will be repeated until $80 \%$ agreement or higher is reached between the reviewers. We will contact the first author of the included studies once, if necessary, for any missing data.

Data items

Once all studies are identified, data extraction will be performed using a standardized data extraction form in Microsoft Excel (Microsoft Corporation, Seattle, Washington, USA). We will collect data related to key study items that include but not limited to variables in Table 1 below. If means and measures of dispersion are reported via figures in the primary studies, they will be approximated using online tools such as Webplotdigitizer (A. Rohatgi, 2020, Pacifica, California, USA).

\section{Outcomes and priorization}

The primary outcome is histological signs of NEC measured at any time after the administration of MSCs. The histological signs can be tissue inflammation, bacterial invasion, tissue necrosis and signs of micro-perforation. If applicable, we will use the standard histologic scoring previously described in the literature to assess the severity of histological changes in NEC $(41,47)$. Histopathology of the lesions will be categorized in four classes: grade 1 focal, mild injury confined to villous types, grade II, partial loss of vili, grade III, necrosis extending to submucosa and grade IV, transmural necrosis.

The secondary outcomes measured will be: survival, bowel perforation, gut permeability, gut motility, levels of inflammatory markers, cytokine levels and any reported adverse events. Survival will be measured in number of days between the diagnosis of NEC and unintentional death. Bowel perforation will be defined as any free air in the abdominal cavity on imaging or visualization of a hole in the wall of the intestinal barrier by direct visualization or by histology. Any possible parameters to assess gut permeability (including but not restricted to disruption of thigh junction proteins, liver fatty acid-binding protein level and intestinal fatty acid binding protein level) and motility will be considered as a valid measure for these outcomes. Any kind of inflammatory markers (including but not restricted to white 
blood cells count (cells $\times 10^{9} / \mathrm{L}$ ), C-reactive protein ( $\mathrm{mg} / \mathrm{L}$ ) and fecal calprotectine $(\mathrm{mcg} / \mathrm{g})$ ) and cytokine levels (including but not restricted to interleukine-8 $(\mathrm{pg} / \mathrm{mL}$ ) and present of toll-like receptor 4 ) will be recorded. All reported adverse events will be considered.

\section{Risk of bias in individual studies}

Two independent investigators ( $\mathrm{CMB}$ and $\mathrm{EH}$ ) will evaluate the risk of bias for each study included in the review. We will use the SYRCLE's risk of bias tool for animal studies (48). This tool was created to establish consistency and avoid discrepancies in the assessment of risk of bias in systematic reviews of animal intervention studies. Each item will be assigned a value of low, high or unclear. Elements of the SYRCLE's tool include assessment of method used for the sequence generation, description of baseline animal characteristics, allocation concealment, random animal housing, blinding of outcome assessment, random outcome assessment, completeness of outcome data and statement of selective outcome reporting.

\section{Data synthesis}

Descriptive statistics and tables of evidence summary will be reported. Study characteristics will be summarized using frequencies and percentages. As a rule, heterogeneity that may be explained by clinical or methodological differences between studies will preclude any planned meta-analyses. If so, study results will be synthesized narratively. Outcomes with dichotomous endpoints (e.g., survival and presence of intestinal perforation) from each included study will be pooled and described using risk ratio and/ or odds ratios and $95 \%$ confidence intervals that incorporate a random effect modelling approach. We will use forest plots to visualize the data. Statistical heterogeneity between studies will be quantified with I-squared statistics. Sparse data will not be meta-analyzed but described narratively. Outcomes with continuous endpoints (E.g., gut permeability, gut motility, levels of inflammatory markers and cytokine levels) will be pooled using mean difference or standardized mean difference. Meta-regression with multiple study level covariates will be attempted when there are at least 10 studies in the body of evidence. Otherwise, clinical and methodological diversity in studies will be explored in pre-specified subgroup and sensitivity analyses. We will analyze subgroups for the following: animal models, sex, experimental model of NEC (e.g., hypercaloric stress, exposure to hypoxia or exposure to hypothermia), administration route of MSC (e.g., intravenous or intraperitoneal), dose of MSC, timing of MSC administration after induction of NEC (less or equal to one hour, greater than one hour to less or equal to four hours, more than four hours or multiple doses), other treatment used and control group. To assess the potential meta-bias and publication bias, we will use the funnel plot as a graphical tool. The quality of reporting of individual preclinical studies will be assessed in accordance with the elements of the ARRIVE (Animal Research: Reporting of In Vivo Experiments) guidelines (49).

\section{Discussion}

NEC is an important inflammatory disease that affects infants and the current treatment is supportive. In order to reduce the mortality and the morbidity associated with this disease, there is a need for new 
treatment targeting the inflammation process and MSCs may be beneficial. To our knowledge, no systematic review of the use of MSCs in preclinical models for NEC has been reported. The data from this review will provide information on whether there is sufficient rigorous evidence to support a clinical trial testing the feasibility and safety of MSC therapy in neonates with NEC. If not, this study will illustrate any gaps in the field which can guide the design of future research studies to address those issues.

\section{List Of Abbreviations}

MSC: mesenchymal stromal cell, NEC: necrotizing enterocolitis.

\section{Declarations}

\section{Ethics approval and consent to participate:}

not applicable.

Consent for publication:

not applicable.

\section{Availability of all the data and materials:}

the datasets used and/or analysed during the current study are available from the corresponding author on reasonable request.

\section{Competing interests:}

the authors declared that they have no competing interest.

\section{Funding:}

not applicable.

\section{Author's contributions:}

all authors conceived the study and contributed to the study design. CMB and EH were responsible for initial drafting, manuscript revisions and data collection. $\mathrm{KC}$ and NA provided expertise in the design of preclinical systematic reviews. BS provided expertise to develop the search strategy and was responsible for conducting the search. KC, NA, EF and BT provided critical revisions and statistical support. EF and BT provided translational biology expertise and provided oversight for planned eligibility criteria and outcome measures. All authors reviewed several drafts of the manuscript and approved the final version.

\section{Acknowledgements:}

not applicable. 


\section{References}

1. Neu J, Walker WA. Necrotizing enterocolitis. N Engl J Med. 2011;364(3):255-64.

2. Bellodas Sanchez J, Kadrofske M. Necrotizing enterocolitis. Neurogastroenterol Motil. 2019;31(3):e13569.

3. Mizrahi A, Barlow O, Berdon W, Blanc WA, Silverman WA. NECROTIZING ENTEROCOLITIS IN PREMATURE INFANTS. J Pediatr. 1965;66:697-705.

4. Meister AL, Doheny KK, Travagli RA. Necrotizing enterocolitis: It's not all in the gut. Exp Biol Med (Maywood). 2020;245(2):85-95.

5. Yu L, Tian J, Zhao X, Cheng P, Chen X, Yu Y, et al. Bowel Perforation in Premature Infants with Necrotizing Enterocolitis: Risk Factors and Outcomes. Gastroenterol Res Pract. 2016;2016:6134187.

6. Horbar JD, Carpenter JH, Badger GJ, Kenny MJ, Soll RF, Morrow KA, et al. Mortality and neonatal morbidity among infants 501 to 1500 grams from 2000 to 2009. Pediatrics. 2012;129(6):1019-26.

7. Stoll BJ, Hansen NI, Bell EF, Shankaran S, Laptook AR, Walsh MC, et al. Neonatal outcomes of extremely preterm infants from the NICHD Neonatal Research Network. Pediatrics. 2010;126(3):44356.

8. Irvine B, Dzakpasu S, León JA. Perinatal health indicators 2013: a surveillance report by the Public Health Agency of Canada's Perinatal Surveillance System. Health Promot Chronic Dis Prev Can. 2015;35(1):23-4.

9. Holman RC, Stoll BJ, Curns AT, Yorita KL, Steiner CA, Schonberger LB. Necrotising enterocolitis hospitalisations among neonates in the United States. Paediatr Perinat Epidemiol. 2006;20(6):498506.

10. Yee WH, Soraisham AS, Shah VS, Aziz K, Yoon W, Lee SK. Incidence and timing of presentation of necrotizing enterocolitis in preterm infants. Pediatrics. 2012;129(2):e298-304.

11. Fitzgibbons SC, Ching Y, Yu D, Carpenter J, Kenny M, Weldon C, et al. Mortality of necrotizing enterocolitis expressed by birth weight categories. J Pediatr Surg. 2009;44(6):1072-5; discussion 5-6.

12. Wang K, Tao G, Sylvester KG. Recent Advances in Prevention and Therapies for Clinical or Experimental Necrotizing Enterocolitis. Dig Dis Sci. 2019;64(11):3078-85.

13. Gordon PV, Swanson JR. Necrotizing enterocolitis is one disease with many origins and potential means of prevention. Pathophysiology. 2014;21(1):13-9.

14. Denning TL, Bhatia AM, Kane AF, Patel RM, Denning PW. Pathogenesis of NEC: Role of the innate and adaptive immune response. Semin Perinatol. 2017;41(1):15-28.

15. Pammi M, De Plaen IG, Maheshwari A. Recent Advances in Necrotizing Enterocolitis Research: Strategies for Implementation in Clinical Practice. Clin Perinatol. 2020;47(2):383-97.

16. Benkoe TM, Mechtler TP, Weninger M, Pones M, Rebhandl W, Kasper DC. Serum levels of interleukin-8 and gut-associated biomarkers in diagnosing necrotizing enterocolitis in preterm infants. J Pediatr Surg. 2014;49(10):1446-51. 
17. Rees CM, Pierro A, Eaton S. Neurodevelopmental outcomes of neonates with medically and surgically treated necrotizing enterocolitis. Arch Dis Child Fetal Neonatal Ed. 2007;92(3):F193-8.

18. Hickey M, Georgieff M, Ramel S. Neurodevelopmental outcomes following necrotizing enterocolitis. Semin Fetal Neonatal Med. 2018;23(6):426-32.

19. Cusick SE, Georgieff MK. The Role of Nutrition in Brain Development: The Golden Opportunity of the "First 1000 Days". J Pediatr. 2016;175:16-21.

20. Moschopoulos C, Kratimenos P, Koutroulis I, Shah BV, Mowes A, Bhandari V. The Neurodevelopmental Perspective of Surgical Necrotizing Enterocolitis: The Role of the Gut-Brain Axis. Mediators Inflamm. 2018;2018:7456857.

21. Lodha A, Asztalos E, Moore AM. Cytokine levels in neonatal necrotizing enterocolitis and long-term growth and neurodevelopment. Acta Paediatr. 2010;99(3):338-43.

22. Shah TA, Meinzen-Derr J, Gratton T, Steichen J, Donovan EF, Yolton K, et al. Hospital and neurodevelopmental outcomes of extremely low-birth-weight infants with necrotizing enterocolitis and spontaneous intestinal perforation. J Perinatol. 2012;32(7):552-8.

23. Bisquera JA, Cooper TR, Berseth CL. Impact of necrotizing enterocolitis on length of stay and hospital charges in very low birth weight infants. Pediatrics. 2002;109(3):423-8.

24. Stey A, Barnert ES, Tseng CH, Keeler E, Needleman J, Leng M, et al. Outcomes and costs of surgical treatments of necrotizing enterocolitis. Pediatrics. 2015;135(5):e1190-7.

25. Knell J, Han SM, Jaksic T, Modi BP. Current Status of Necrotizing Enterocolitis. Curr Probl Surg. 2019;56(1):11-38.

26. Friedenstein AJ, Gorskaja JF, Kulagina NN. Fibroblast precursors in normal and irradiated mouse hematopoietic organs. Exp Hematol. 1976;4(5):267-74.

27. Gu Y, Zhang Y, Bi Y, Liu J, Tan B, Gong M, et al. Mesenchymal stem cells suppress neuronal apoptosis and decrease IL-10 release via the TLR2/NFKB pathway in rats with hypoxic-ischemic brain damage. Mol Brain. 2015;8(1):65.

28. Mueller M, Oppliger B, Joerger-Messerli M, Reinhart U, Barnea E, Paidas M, et al. Wharton's Jelly Mesenchymal Stem Cells Protect the Immature Brain in Rats and Modulate Cell Fate. Stem Cells Dev. 2017;26(4):239-48.

29. Donega V, Nijboer CH, Braccioli L, Slaper-Cortenbach I, Kavelaars A, van Bel F, et al. Intranasal administration of human MSC for ischemic brain injury in the mouse: in vitro and in vivo neuroregenerative functions. PLoS One. 2014;9(11):e112339.

30. Ahn SY, Chang YS, Sung DK, Sung SI, Yoo HS, Lee JH, et al. Mesenchymal stem cells prevent hydrocephalus after severe intraventricular hemorrhage. Stroke. 2013;44(2):497-504.

31. Zhu LH, Bai X, Zhang N, Wang SY, Li W, Jiang L. Improvement of human umbilical cord mesenchymal stem cell transplantation on glial cell and behavioral function in a neonatal model of periventricular white matter damage. Brain Res. 2014;1563:13-21. 
32. Wehman B, Sharma S, Pietris N, Mishra R, Siddiqui OT, Bigham G, et al. Mesenchymal stem cells preserve neonatal right ventricular function in a porcine model of pressure overload. Am J Physiol Heart Circ Physiol. 2016;310(11):H1816-26.

33. Aslam M, Baveja R, Liang OD, Fernandez-Gonzalez A, Lee C, Mitsialis SA, et al. Bone marrow stromal cells attenuate lung injury in a murine model of neonatal chronic lung disease. Am J Respir Crit Care Med. 2009;180(11):1122-30.

34. van Haaften T, Byrne R, Bonnet S, Rochefort GY, Akabutu J, Bouchentouf M, et al. Airway delivery of mesenchymal stem cells prevents arrested alveolar growth in neonatal lung injury in rats. Am J Respir Crit Care Med. 2009;180(11):1131-42.

35. McCulloh CJ, Olson JK, Wang Y, Vu J, Gartner S, Besner GE. Evaluating the efficacy of different types of stem cells in preserving gut barrier function in necrotizing enterocolitis. J Surg Res. 2017;214:27885.

36. McCulloh CJ, Olson JK, Zhou Y, Wang Y, Besner GE. Stem cells and necrotizing enterocolitis: A direct comparison of the efficacy of multiple types of stem cells. J Pediatr Surg. 2017;52(6):999-1005.

37. Wang JD, An Y, Zhang JS, Wan XH, Jonas JB, Xu L, et al. Human bone marrow mesenchymal stem cells for retinal vascular injury. Acta Ophthalmol. 2017;95(6):e453-e61.

38. Bernardo ME, Pagliara D, Locatelli F. Mesenchymal stromal cell therapy: a revolution in Regenerative Medicine? Bone Marrow Transplant. 2012;47(2):164-71.

39. Shamseer L, Moher D, Clarke M, Ghersi D, Liberati A, Petticrew M, et al. Preferred reporting items for systematic review and meta-analysis protocols (PRISMA-P) 2015: elaboration and explanation. Bmj. 2015;350:g7647.

40. de Vries RB, Hooijmans CR, Tillema A, Leenaars M, Ritskes-Hoitinga M. A search filter for increasing the retrieval of animal studies in Embase. Lab Anim. 2011;45(4):268-70.

41. Mendez YS, Khan FA, Perrier GV, Radulescu A. Animal models of necrotizing enterocolitis. World Journal of Pediatric Surgery. 2020;3(1):e000109.

42. Kovler ML, Sodhi CP, Hackam DJ. Precision-based modeling approaches for necrotizing enterocolitis. Dis Model Mech. 2020;13(6).

43. Dominici M, Le Blanc K, Mueller I, Slaper-Cortenbach I, Marini F, Krause D, et al. Minimal criteria for defining multipotent mesenchymal stromal cells. The International Society for Cellular Therapy position statement. Cytotherapy. 2006;8(4):315-7.

44. McGowan J, Sampson M, Salzwedel DM, Cogo E, Foerster V, Lefebvre C. PRESS Peer Review of Electronic Search Strategies: 2015 Guideline Statement. J Clin Epidemiol. 2016;75:40-6.

45. Hooijmans CR, Tillema A, Leenaars M, Ritskes-Hoitinga M. Enhancing search efficiency by means of a search filter for finding all studies on animal experimentation in PubMed. Lab Anim. 2010;44(3):170-5.

46. de Vries RB, Hooijmans CR, Tillema A, Leenaars M, Ritskes-Hoitinga M. Updated version of the Embase search filter for animal studies. Lab Anim. 2014;48(1):88. 
47. Caplan MS, Hedlund E, Adler L, Hsueh W. Role of asphyxia and feeding in a neonatal rat model of necrotizing enterocolitis. Pediatr Pathol. 1994;14(6):1017-28.

48. Hooijmans CR, Rovers MM, de Vries RBM, Leenaars M, Ritskes-Hoitinga M, Langendam MW. SYRCLE's risk of bias tool for animal studies. BMC Medical Research Methodology. 2014;14(1):43.

49. Percie du Sert N, Hurst V, Ahluwalia A, Alam S, Avey MT, Baker M, et al. The ARRIVE guidelines 2.0: Updated guidelines for reporting animal research. PLOS Biology. 2020;18(7):e3000410.

\section{Table}


Table 1

Data collection items

\section{Data category Specific items}

Study characteristics
Title, name of corresponding author, number of authors, publication date, journal of publication, study design, single vs. multi-centre trial, funding and country of publication of the corresponding author

Study population

Experimental model of necrotizing enterocolitis

Intervention

Comparison

Type and preparation of control suspension, time of treatment after the diagnosis of NEC, unit, duration, frequency, route of administration, strategy of administration, and co-intervention, etc.

CoFluids, bowel rest, antibiotics, etc.

interventions

Preclinical endpoints

Histological description of intestinal tissue injury, survival, evidence of gut perforation, gut permeability (e.g., disruption of thigh junction proteins, liver fatty acid-binding protein level and intestinal fatty acid binding protein level), gut motility, level of inflammatory markers (e.g., white blood cells count, C-reaction protein and fecal calprotectine), level of cytokines (e.g., interleukine-8 and toll-like receptor 4) and any reported adverse events.

Risk of bias assessments

In accordance with the elements of the SYRCLE's risk of bias tool for animal studies (48): Random sequence generation, description of baseline animal characteristics, allocation concealment, random animal housing blinding of personnel, blinding of outcome assessment, completeness of outcome data and statement of selective reporting.

Quality of reporting of individual preclinical studies

In accordance with the elements of the ARRIVE guidelines (49)

Table 1: Included data collection items related to key study items.

\section{Supplementary Files}

This is a list of supplementary files associated with this preprint. Click to download. 
- SPIDERMNNECMSCsStrategyDraft20210208.docx 